\title{
Soziale Arbeitsbedingungen der Schweizer Lehrpersonen - Ergebnisse einer nationalen Studie
}

\section{Sophie Baeriswyl, Doris Kunz Heim und Andreas Krause}

Basierend auf einer landesweiten Befragung werden die sozialen Arbeitsbedingungen von Schweizer Lehrpersonen sowie potentielle Unterschiede hinsichtlich Demographie (Geschlecht, Berufserfahrung und Sprachregion) und Schulgrösse betrachtet. Die Ergebnisse deuten darauf hin, dass Schweizer Lehrpersonen ihre sozialen Arbeitsbedingungen im Vergleich zu einer Referenzstichprobe aus Baden-Württemberg (Bundesland in Deutschland) mehrheitlich positiv bewerten. Hinsichtlich der Sprachregion zeigten sich signifikante Unterschiede. Die Untersuchungsergebnisse werden mit Blick auf die Aus- und Weiterbildung von Schweizer Lehrpersonen diskutiert.

\section{Einleitung und theoretischer Rahmen}

Die Lehrerbelastung und -beanspruchung ist eine aktuelle und brisante Thematik sowohl in der öffentlichen Diskussion als auch in der Schul- und Bildungsforschung (Cramer, Merk \& Wesselborg, 2014; Rothland, 2013a; Rothland \& Klusmann, 2012). Wir gehen davon aus, dass Veränderungen bei der Steuerung des Bildungssystems dazu beitragen, dass Fragen der Arbeitsorganisation an der einzelnen Schule und ihrer Auswirkungen auf die Beteiligten (z.B. Belastungen bei der Zusammenarbeit) an Bedeutung zunehmen (Krause \& Dorsemagen, 2011). Grundsätzlich sind die Veränderungen im Bildungssystem gekennzeichnet durch einen Übergang von der Inputsteuerung (charakterisiert z.B. durch exakt definierte Lehrpläne und Prüfungsrichtlinien) zur Outputsteuerung (charakterisiert z.B. durch eine verstärkte Ausrichtung der Bildungspolitik an den Leistungen und Lernergebnissen der Schülerschaft), was in der Schweiz als wirkungsorientierte Verwaltungsführung bezeichnet wird. Die einzelnen Schulen erhalten bei konsequenter Umsetzung mehr Freiheiten bzw. Teilautonomie (z.B. über Globalbudgets), um eigenverantwortlich festzulegen, wie die Outputkriterien am besten erreicht werden können. Diese Entwicklungen gehen mit einer Forderung nach mehr und v.a. qualitativ hochwertiger Kooperation in 
den Schulteams einher, haben aber auch Auswirkungen auf die Belastungen und Beanspruchungen von Lehrpersonen (Morgenroth, 2015). Wir werden im Laufe des Beitrages wiederholt auf diese Thematik zurückkommen.

Was ist mit Belastung und Beanspruchung genau gemeint? Wir beziehen uns bei der Definition und der begrifflichen Trennung von Belastung und Beanspruchung auf das in Abbildung 1 ersichtliche theoretische Rahmenmodell schulischer Belastung und Beanspruchung von Böhm-Kasper (2004). Demnach sind Belastungen die Gesamtheit der erfassbaren äußeren, auf den Menschen einwirkenden Einflüsse, von denen man annehmen kann, dass sie eine gewisse Anstrengung zu ihrer Bewältigung erfordern. Demgegenüber kennzeichnet die psychische Beanspruchung die Auswirkung dieser Belastung auf den Menschen in Abhängigkeit von den individuellen Voraussetzungen. Mit dieser Begriffsdifferenzierung wird das Modell der Tatsache gerecht, dass Menschen individuell verschieden auf identische äußere Einflüsse reagieren (Böhm-Kasper \& Weishaupt, 2002). Dieser Umstand wird im Modell mit dem Begriff des subjektiven Deutungsprozesses verdeutlicht. In der vorliegenden Studie stehen die in Abbildung 1 schwarz dargestellten Bestandteile des Modells, sprich die Belastungsfaktoren von Schweizer Lehrpersonen, im Zentrum. Die grau dargestellten Bereiche der subjektiven Deutungsprozesse und Beanspruchung sind nicht Teil dieses Beitrages.

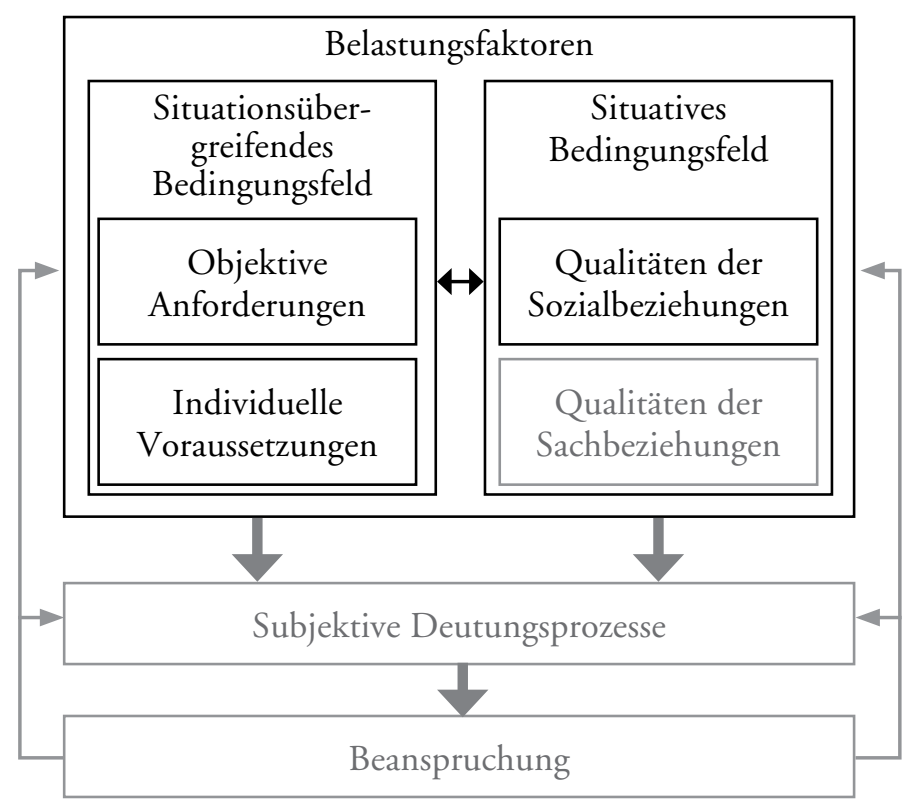

Abbildung 1: Modell zur schulischen Belastung und Beanspruchung (in Anlehnung an Böhm-Kasper \& Weishaupt, 2012, S. 476) 
Der Belastungsbegriff ist im Rahmenmodell schulischer Belastung neutral formuliert. Das impliziert, dass Belastungen sowohl negativ (Fehlbelastung, Stressor) wie auch positiv (Entlastungs- bzw. Unterstützungsfaktor, Ressource) erlebt werden und entsprechend sowohl Distress wie auch Eustress auslösen können. Die Belastungssituation an einer Schule wird neben der Qualität der Sachbeziehungen (wie z.B. die Reaktion der Schüler auf die im Unterricht vermittelten Inhalte) vor allem durch die Qualität der Sozialbeziehungen bestimmt (Böhm-Kasper \& Weishaupt, 2002). Diese stehen in unserer Untersuchung im Zentrum.

\section{Die Qualität der Sozialbeziehungen als zentraler \\ Belastungsfaktor im situativen Bedingungsfeld}

Die Arbeitssituation an Schulen ist vielfach sozial geprägt, über die Interaktionen zwischen den Lehrpersonen und der Schülerschaft, über die Kooperation unter den Lehrerinnen und Lehrern und selbstverständlich auch über die Schulleitungen. Die Qualität dieser sozialen Beziehungen ist von grosser Bedeutung für das Belastungserleben, die pädagogische Autonomie und letztlich für die Lehrergesundheit (Rothland, 2013b). Die konkret stattfindende Kooperation und Zusammenarbeit in Schulen ist an spezifische Situationen und Aufgaben gebunden (Dizinger, Fussangel \& Böhm-Kasper, 2011). Deshalb wird sie im vorliegenden Beitrag dem situativen Bedingungsfeld in Abbildung 1 zugeordnet.

Wenn von der Qualität der Sozialbeziehungen die Rede ist, steht häufig die Kooperation zwischen Lehrpersonen untereinander im Zentrum (Trumpa, Franz \& Greiten, 2016). Fussangel, Dizinger, Böhm-Kasper und Gräsel (2010) differenzieren drei Intensitätsgrade der Kooperation, von denen lediglich die intensivste Form, die sogenannte Ko-Konstruktion (als das gemeinsame Entwickeln von Standards und neuen Wissens), von Lehrpersonen als Entlastung wahrgenommen wird, sprich mit einem niedrigeren Belastungserleben in Verbindung steht (Dizinger et al., 2011), obwohl gerade bei Ko-Konstruktion zunächst Arbeitszeit investiert werden muss. Dieser Befund ist möglicherweise dadurch zu erklären, dass positive Zusammenhänge bestehen zwischen sozialer Unterstützung und Lehrerkooperation, sofern eine positive Gewinnspirale zum Aufbau von Ressourcen führt (Morgenroth, 2015). Im Rahmen intensiver Kooperation sind also eher soziale Unterstützungsprozesse möglich, sofern eine erfolgreiche gemeinsame Interaktion gelingt. Gemeinsame pädagogische Vorstellungen sind ein Ergebnis von Ko-Konstruktion, d.h. gemeinsame handlungsleitende Ziele entstehen insbesondere über das gemeinsame Vorbereiten, Durchführen und Nachbereiten von Unterricht (Halfhide, 2009).

Die Qualität der Sozialbeziehungen an Schulen ist nicht ausschliesslich von der Kooperation unter Lehrpersonen und dem damit verbundenen Geben und Erhalten sozialer Unterstützung abhängig, sondern beinhaltet auch Kooperation zwischen Lehrkräften auf der einen Seite und Schülerschaft, Eltern und anderen schulischen Akteuren auf der anderen Seite. Von besonderer Bedeutung ist die 
Qualität der Lehrperson-Schüler-Beziehung. Als charakteristisches Merkmal des Lehrerberufs hebt Rothland (2013c) «die erzwungene Zusammenarbeit und das asymmetrische Verhältnis zwischen den Schülern und den Lehrkräften» (S. 23) hervor und weiter: «Lehrerinnen und Lehrer befinden sich im Rahmen ihrer primären Tätigkeit - dem Unterrichten - in einer sozialen Konstellation mit einer Gruppe von Schülerinnen und Schülern, die i.d.R. nicht auf Freiwilligkeit beruht» (S. 25). Entsprechend sehen wir Störungen im Unterricht als bedeutsame soziale Belastung an. Gleichzeitig beinhaltet die Beziehung zwischen Lehrkräften und Schülern auch für Lehrkräfte selbst viel unterstützendes Potential, z.B. in Form von Gratifikation durch Anerkennung. Dies erscheint wesentlich für ein Verständnis, warum Lehrpersonen im Vergleich zu anderen Berufsgruppen keineswegs weniger Anerkennung berichten (Schult, Münzer-Schrobildgen \& Sparfeldt, 2014). Deshalb berücksichtigen wir auch die Anerkennung bzw. Unterstützung durch Schüler.

Im Vergleich zur Interaktion mit Schülerinnen und Schülern ist der zeitliche Anteil der Interaktion mit Eltern deutlich geringer (Philipp \& Kunter, 2013). In einer Schweizer Befragung sahen sich gleichwohl 40\% der Lehrpersonen gestresst aufgrund der hierfür notwendigen Zeit (Schaller, 2015). Noch bedeutsamer als die Zeit wird die Qualität der Beziehung sein: In norwegischen Studien zeigte sich, dass Konflikte mit Eltern mit erhöhter emotionaler Erschöpfung einhergingen (Skaalvik \& Skaalvik, 2007), während positive soziale Beziehungen mit Eltern Erschöpfung reduzieren können (Skaalvik \& Skaalvik, 2010), so dass neben Konflikten auch Unterstützung durch Eltern berücksichtigt werden sollte.

In den Bildungsreformen der letzten zwanzig bis dreissig Jahren wurde die Bedeutung der Schulleitungen gestärkt, insbesondere in der Schweiz. Vor diesem Hintergrund überrascht es nicht, dass gewonnene Erkenntnisse zur Bedeutung der Führungsqualität für die Gesundheit der Mitarbeitenden (Gregersen, Zimber, Kuhnert \& Nienhaus, 2011) zunehmend auch für Lehrerinnen und Lehrer nachgewiesen werden (z.B. Laux, 2011).

Neben situativen (also die Einzelschule betreffenden Einflüssen werden im Modell von Böhm-Kasper (2004) auch situationsübergreifende Faktoren als Belastungsfaktoren diskutiert.

\section{Der Einfluss objektiver Anforderungen und individu- eller Voraussetzungen}

Belastungsfaktoren werden nicht nur durch die Qualität der Sozialbeziehungen konstituiert, sondern auch durch situationsübergreifende Faktoren. Dazu gehören einerseits Belastungen, deren Ursachen systembedingt sind (objektive Anforderungen), und andererseits Belastungen, die durch individuelle Dispositionen der Lehrerpersonen bestimmt werden (individuelle Voraussetzungen). Diese situationsübergreifenden Belastungsfaktoren können nicht nur die subjektiven Deutungsprozesse und in der Folge die Beanspruchung von Lehrpersonen, sondern auch die Qualität der Sozialbeziehungen beeinflussen, wie auch im 
theoretischen Rahmenmodell schulischer Belastung und Beanspruchung durch den Doppelpfeil in Abbildung 1 angedeutet wird.

\section{Sprachregionale Gegebenheiten und Schulgrösse als objektive Anforderungen} im situationsübergreifenden Bedingungsfeld

Zum situationsübergreifenden Bedingungsfeld gehören schulinstitutionelle Voraussetzungen wie die Schulgrösse (Böhm-Kasper, 2004), da diese Auswirkungen auf die Arbeitsbedingungen von Lehrpersonen haben kann (Dizinger, Fussangel \& Böhm-Kasper, 2011). Mit zunehmender Größe von Arbeitszusammenhängen steigt der Koordinations- und Absprachebedarf überproportional an (Forneck \& Schriever, 2000). Entsprechend schwieriger wird es, im Rahmen des Leitungshandelns Transparenz herzustellen, was die Zusammenarbeit mit der Schulleitung, im Kollegium, mit den Eltern und mit der Schülerschaft erschweren kann. Auch die Ergebnisse internationaler empirischer Studien deuten darauf hin, dass Lehrpersonen von kleinen Schulen weniger stark belastet sind als dies bei Lehrpersonen von grossen Schulen der Fall ist (Cannata, 2007; Harazd, Gieske, Gerick \& Rolff, 2009; Nübling, Vomstein, Haug, Nübling \& Adiwidjaja, 2011). In der Schweiz wurde der Einfluss der Schulgrösse auf die Qualität der Sozialbeziehungen noch nicht systematisch erforscht.

Eine Schweizer Eigenheit ist die Bedeutung unterschiedlicher Sprachregionen. Igic et al. (2015) konnten in einer für Schweizer Erwerbstätige repräsentativen Stichprobe aufzeigen, dass die Arbeitsbedingungen in den französischund italienischsprachigen Regionen leicht kritischer bewertet werden als in der Deutschschweiz. Es liegen erste Hinweise vor, dass dies auch für Erwerbstätige im Bildungssystem zutreffend ist (Sandmeier, Kunz Heim, Windlin \& Krause, 2017). Kantonale und regionale Unterschiede in den Bildungssystemen erhöhen die Wahrscheinlichkeit, dass Lehrpersonen aus unterschiedlichen Sprachregionen ihre sozialen Arbeitsbedingungen unterschiedlich beurteilen (z.B. ist die Harmonisierung der Lehrpläne in den Westschweizer Kantonen früher vorangetrieben worden). Den Autoren ist allerdings keine Untersuchung bekannt, in welcher Unterschiede hinsichtlich der sozialen Belastungen zwischen den Sprachregionen systematisch betrachtet wurden.

\section{Geschlecht und Berufserfahrung als individuelle Voraussetzungen im situa- tionsïbergreifenden Bedingungsfeld}

Untersuchungsergebnisse zum Einfluss individueller Voraussetzungen wie Geschlecht und Berufserfahrung sind oft widersprüchlich. So zeigten zum Beispiel viele empirische Untersuchungen höhere Werte bei subjektiv erlebtem Stress bei Lehrerinnen als bei Lehrern (Antoniou, Polychroni \& Vlachakis, 2006; Chaplain, 2008; Klassen \& Chiu, 2010), andere jedoch fanden keinen Geschlechtereffekt (Schult, Münzer-Schrobildgen \& Sparfeldt, 2014). Die Ergebnisse von Vuille, Carvajal, Casaulta und Schenkel (2004) deuten im Gegensatz dazu auf eine geringere Belastung von Lehrerinnen im Vergleich zu ihren männlichen 
Kollegen hin. Mehrere Untersuchungsergebnisse sprechen zudem dafür, dass Unterschiede zwischen Lehrpersonen mit unterschiedlicher Berufserfahrung nicht bedeutsam sind (Forneck \& Schriever, 2001; Grayson \& Alvarez, 2008; Schult et al., 2014). Demgegenüber stehen wiederum die Ergebnisse von Vuille et al. (2004), wo Junglehrer der Stadt Bern die geringste Belastung zeigten.

\section{Fragestellungen}

Die erste Fragestellung lautete: Wie bewerten Schweizer Lehrpersonen die sozialen Arbeitsbedingungen im Vergleich zu einer Referenzstichprobe aus Baden-Württemberg?

Als relevante soziale Arbeitsbedingungen wurden die Kooperation mit Kolleginnen und Kollegen (gemeinsame pädagogische Vorstellungen), mit Schülerinnen und Schülern (Unterstützung durch Schüler, Störungen im Unterricht), mit Eltern (Unterstützung durch Eltern, Konflikte mit Eltern) sowie mit der Schulleitung (Führungsqualität) berücksichtigt. Zur Interpretation der Ergebnisse dienten bei fünf der sechs sozialen Arbeitsbedingungen Vergleiche mit einer Vollerhebung von Lehrkräften aus Baden-Württemberg (Bundesland in Deutschland).

Die zweite Fragestellung lautete: Unterscheiden sich die sozialen Arbeitsbedingungen von Lehrpersonen nach Sprachregionen, Schulgrösse, Berufserfahrung oder Geschlecht?

Als situationsübergreifende Bedingungen wurden sowohl objektive Anforderungen (Schulgrösse, Sprachregion) als auch individuelle Voraussetzungen (Geschlecht, Berufserfahrung) berücksichtigt.

\section{Methode}

\section{Studie und Stichprobe}

Der vorliegende Beitrag wurde im Rahmen der vom Schweizerischen Nationalfonds (SNF) unterstützen Studie "Ressourcen und Belastungen von Schweizer Lehrpersonen» (RBSL) verfasst. Die Rekrutierung der Probanden sowie die Datenerhebung erfolgte im ersten Quartal 2010 durch Sucht Schweiz (ehemals Schweizerische Fachstelle für Alkohol- und andere Drogenprobleme). Sucht Schweiz führt unter der Schirmherrschaft der Weltgesundheitsorganisation WHO alle vier Jahre eine Studie zum Gesundheitsverhalten von Schweizer Schülerinnen und Schülern zwischen 11 und 15 Jahren durch (Health Behaviour in School-Aged Children [HBSC]). Seit 2002 erhalten auch die Lehrpersonen der befragten Klassen einen Fragebogen, mit dem Daten zur Belastungssituation und der Verfügbarkeit von Ressourcen erhoben werden. Basierend auf dem Verzeichnis aller Klassen des 5. bis 9. Schuljahres der öffentlichen Schulen des Bundesamtes 
für Statistik erfolgte eine randomisierte Auswahl von 714 Klassen und ihren Klassenlehrpersonen. Der Fragebogen wurde von 586 Lehrpersonen ausgefüllt und zurückgesandt, was einer Rücklaufquote von 83 Prozent entspricht.

Von den 586 befragten Lehrpersonen waren 261 (45\%) weiblich. Ein Grossteil der Lehrpersonen (459; 78\%) war zum Zeitpunkt der Befragung zwischen 30 und 50 Jahre alt. 32 Lehrpersonen (6\%) waren 60 Jahre alt oder älter und 95 Lehrpersonen (16\%) waren 29 Jahre alt oder jünger. 171 Lehrpersonen (29\%) hatten zum Zeitpunkt der Befragung mehr als 25 Jahre Berufserfahrung; 168 Lehrpersonen (29\%) 13 bis 25 Jahre; 126 Lehrpersonen (22\%) 6 bis 12 Jahre; und 121 Lehrpersonen (21\%) 5 Jahre oder weniger. 420 der befragten Lehrpersonen (72 \%) stammten aus der Deutschschweiz, 166 Lehrpersonen (28\%) aus der französisch- und italienischsprachigen Schweiz. Die meisten Lehrpersonen (221, $38 \%$ ) unterrichteten an Schulen mittlerer Grösse (21-40 Lehrpersonen/ Schule). 170 (29\%) waren an kleinen Schulen ( $\leq 20$ Lehrpersonen/Schule) und $189(32 \%)$ an grossen Schulen ( $\geq 41$ Lehrpersonen/Schule) tätig. 6 Lehrpersonen (1\%) machten keine Angabe zur Grösse ihrer Schule.

Es handelt sich um eine auf der Ebene der Klassen des 5. bis 9. Schuljahres der öffentlichen Schulen für die Schweiz repräsentative Stichprobe. Auf der Ebene der Lehrpersonen ist die Stichprobe nicht repräsentativ für die Schweiz. Einschränkungen Repräsentativität ergeben sich insbesondere hinsichtlich der Funktion (Klassenlehrpersonen sind in der Stichprobe überrepräsentiert, da der Fragebogen direkt an sie gerichtet war) und unmittelbar damit zusammenhängend auch hinsichtlich des Geschlechts (weibliche Lehrpersonen sind in der Stichprobe unterrepräsentiert) und des Unterrichtspensums (Lehrpersonen mit Kleinpensen sind in der Stichprobe unterrepräsentiert).

Die Werte der vorliegenden Untersuchung konnten wir mit Angaben aus Baden-Württemberg (Deutschland) vergleichen. Diese Werte basieren auf den Angaben von über 50'000 Lehrkräften aus Baden-Württemberg, welche in acht Tranchen im Zeitraum von 2008 bis 2010 im Rahmen einer Vollerhebung befragt wurden (Nübling et al., 2012).

\section{Erhebungsinstrumente}

Die sozialen Arbeitsbedingungen von Schweizer Lehrpersonen haben wir mit den Skalen des Copenhagen Psychosocial Questionnaire-Fragebogen zur Arbeitssituation an Schulen (COPSOQ-FASS) erfasst (Krause, 2004; Nübling et al., 2012; Nübling, Wirtz, Neuner \& Krause, 2008). Sämtliche Items wurden auf einer Likert-Skala mit den Polen 1 (trifft nicht zu / in sehr geringem Mass) und 5 (trifft sehr zu / in sehr hohem Mass) beurteilt. Mit Ausnahme der von Doris Kunz Heim und Anita Sandmeier neu entwickelten Skala Unterstützung durch Schüler wurden die in diesem Beitrag verwendeten Skalen im Rahmen der Personenbezogenen Gefährdungsbeurteilung an öffentlichen Schulen in Baden-Württemberg eingesetzt und hinsichtlich ihrer Faktorenstruktur und Skalenreliabilität bereits geprüft. Demnach wiesen die Skalen gute bis sehr gute 
Messeigenschaften auf (Nübling et al., 2012). In der Folge beschränken wir uns deshalb auf die Darstellung von Cronbach's $\alpha$ als Indikator für die Skalenreliabilität. Bei der Skala Unterstützung durch Schüler, die nicht Bestandteil der Befragung in Baden-Württemberg war, haben wir zusätzlich die Faktorenstruktur im Rahmen einer konfirmatorischen Faktorenanalyse betrachtet. Mittelwerte, Standardabweichungen und Interkorrelationen finden sich in Tabelle 1.

Tabelle 1: Mittelwerte (M) und Standardabweichungen (SD) für die Schweiz $(N=586)$ sowie Pearson Korrelationen zwischen den Skalen

\begin{tabular}{lccccccc}
\hline \multicolumn{1}{c}{ Schweiz } & & & & & \\
& $M$ & $S D$ & 1 & 2 & 3 & 4 & 5 \\
\hline 1 Gemeinsame pädagogische Vorstellungen & 3.37 & 0.78 & - & & & & \\
2 Unterstützung durch Schüler & 3.66 & 0.61 & $.24^{* * *}$ & - & & & \\
3 Störungen im Unterricht & 2.58 & 0.68 & $-.22^{* * *}$ & $-.44^{* * *}$ & - & & \\
4 Unterstützung durch Eltern & 3.42 & 0.67 & $.31^{* * *}$ & $.41^{* * *}$ & $-.34^{* * *}$ & - & \\
5 Konflikte mit Eltern & 2.26 & 0.81 & $-.19^{* * *}$ & $-.32^{* * *}$ & $.28^{* * *}$ & $-.40^{* * *}$ & \\
6 Führungsqualität & 3.56 & 0.78 & $.38^{* * *}$ & $.15^{* * *}$ & -.05 & $.15^{* * *}$ & -.06 \\
\hline
\end{tabular}

${ }^{* * *} p \leq .001$.

Die Lehrerkooperation haben wir mit Hilfe der aus fünf Items zusammengesetzten Skala Gemeinsame pädagogische Vorstellungen (z.B. «In Erziehungs- und Disziplinfragen ziehen alle Kollegen/Kolleginnen an einem Strang») operationalisiert. Cronbach's $\alpha$ betrug .88. Die Entwicklung und Aufrechterhaltung gemeinsamer pädagogischer Vorstellungen kann als intensive Form der Lehrerkooperation betrachtet werden (Morgenroth, 2015). Zur Beschreibung der Interaktionen mit der Schülerschaft haben wir die aus drei Items bestehende Skala Unterstützung durch Schüler («Man wird von den Schülern akzeptiert und anerkannt»; "Man wird in seiner Arbeit durch die Schüler unterstützt»; «Die Schüler halten sich an Absprachen») und die aus sieben Items bestehende Skala Störungen im Unterricht (z.B. «Im Unterricht sind ständig Ermahnungen notwendig») verwendet. Die Ergebnisse der konfirmatorischen Faktorenanalyse unterstützen die Zusammenfassung der drei obengenannten Items zur Skala Unterstützung durch Schüler: Alle drei Indikatoren hatten signifikante Faktorladungen $(p<.05)$. Diese waren höher als der von Hair, Black, Babin und Anderson (2014) propagierte Mindestwert von .50. Cronbach's $\alpha$ dieser Skala betrug .63, Cronbach's $\alpha$ der Skala Störungen im Unterricht betrug .81.

Die Interaktionen mit den Eltern haben wir mittels zweier Skalen erhoben, zum einen mit der aus drei Items bestehenden Skala Unterstützung durch Eltern (z.B. «Bei Schülerproblemen kann man mit den Eltern zusammenarbeiten») und zum anderen mit der ebenfalls drei Items umfassenden Skala Konflikte mit 
Eltern (z.B. «Die Eltern der Schülerinnen und Schüler kritisieren die Arbeit der Lehrkräfte»). Cronbach's $\alpha$ betrug .78 respektive .89 .

Schliesslich haben wir die aus vier Items bestehende Skala Führungsqualität (z.B. «Bitte schätzen Sie ein, in welchem Mass Ihr unmittelbarer Vorgesetzter / Ihre unmittelbare Vorgesetzte für gute Entwicklungsmöglichkeiten der einzelnen Lehrpersonen sorgt» zur Beschreibung der Interaktionen mit und der Unterstützung durch die Schulleitung verwendet. Cronbach's $\alpha$ betrug .85.

\section{Analyseme thode}

Im Hinblick auf die Fragestellung 1 haben wir Mittelwerte und Standardabweichungen der Skalen in SPSS (Version 23) berechnet. Die Werte der vorliegenden Untersuchung haben wir mit Referenzwerten aus Baden-Württemberg verglichen und mittels t-Tests auf statistische Signifikanz geprüft. Um einer Kumulierung der $\alpha$-Fehlerrate entgegenzuwirken, haben wir das Signifikanz-Niveau gemäss Bonferroni ( $\alpha=.05$ / Anzahl Vergleiche; Abdi, 2007) angepasst.

Im Hinblick auf die Fragestellung 2 haben wir im Rahmen einer multivariaten Varianzanalyse (MANOVA) unter Verwendung der Pillai-Bartlett Trace Teststatistik Mittelwertunterschiede in den sozialen Arbeitsbedingungen von Schweizer Lehrpersonen hinsichtlich Geschlecht, Berufserfahrung, Sprachregion und Schulgrösse (erfasst über die Anzahl Lehrpersonen an der Schule) analysiert. Signifikante multivariate Testergebnisse haben wir im Rahmen von einfaktoriellen Varianzanalysen (ANOVAs) (bei mehr als zwei Gruppen) oder t-Tests (bei zwei Gruppen) genauer betrachtet. Die Signifikanz der entsprechenden Analysen haben wir wiederum basierend auf dem gemäss Bonferroni (Abdi, 2007) angepassten Signifikanzniveau beurteilt.

Da bei grossen Stichproben auch unbedeutende Mittelwertunterschiede signifikant werden können, haben wir bei signifikanten Testergebnissen mit dem Cohen's $d$ und dem partiellen Eta Quadrat zusätzlich Effektstärken berechnet. Diese erlauben es, die Bedeutsamkeit der Mittelwertunterschiede unabhängig vom Stichprobenumfang zu beurteilen. Als bedeutsam erachten wir Mittelwertunterschiede mit einem mindestens mittelgrossen Effekt, das heisst ein Cohen's $d$ ab .50 und ein partielles Eta Quadrat ab .06 (Cohen, 1988).

\section{Resultate}

Belastungen aus den sozialen Arbeitsbedingungen

(Fragestellung 1)

Tabelle 2 zeigt die Resultate der Mittelwertvergleiche zwischen der Schweizer und der Baden-Württemberger Stichprobe. Die Signifikanz der t-Tests zum Vergleich der Schweizer und der Baden-Württemberger Mittelwerte haben wir basierend auf dem gemäss Bonferroni angepassten Signifikanzniveau von $p \leq .01$ (.05 / 5 Vergleiche) beurteilt. 
Bei fünf der sechs Skalen konnte ein Vergleich der Stichproben gemacht werden. Drei Skalen unterscheiden sich signifikant zwischen der Schweizer und der Baden-Württemberger Stichprobe. Die Schweizer Lehrerschaft nahm signifikant weniger Störungen im Unterricht wahr (mittelstarker Effekt), berichtet über signifikant mehr Unterstützung durch Eltern (kleiner bis mittelstarker Effekt) und beurteilt die Führungsqualität signifikant positiver (kleiner Effekt) als die Referenzstichprobe aus Baden-Württemberg. In den Skalen Gemeinsame Pädagogische Vorstellung und Konflikte mit den Eltern unterschieden sich die Stichproben nicht voneinander.

Tabelle 2: Mittelwerte (M) und Standardabweichungen (SD) der Schweizer ( $N$ = 586) und der Baden-Württemberger $(B W)(N=50$ '399-53'377) Stichprobe und Ergebnisse des Mittelwertvergleichs mittels $t$-Tests

\begin{tabular}{lcccccc}
\hline \multicolumn{7}{c}{ Schweiz } \\
& $M$ & $S D$ & $M$ & $S D$ & $t(\mathrm{df})$ & $d$ \\
\hline Gemeinsame pädagogische & 3.37 & 0.78 & 3.29 & 0.86 & $2.17(53955)$ & \\
Vorstellungen & 2.58 & 0.68 & 3.03 & 0.85 & $12.72(53958)^{*}$ & .58 \\
Störungen im Unterricht & 3.42 & 0.67 & 3.20 & 0.66 & $7.91(53699)^{*}$ & .33 \\
Unterstützung durch Eltern & 2.26 & 0.81 & 2.30 & 0.85 & $1.16(53821)$ & \\
Konflikte mit Eltern & 3.56 & 0.78 & 3.35 & 0.91 & $5.55(50970)^{*}$ & .25 \\
Führungsqualität & & & & & & \\
\hline
\end{tabular}

${ }^{*} p \leq .01(.05 / 5$ Vergleiche $)$

\section{Unterschiede zwischen Teilgruppen von Schweizer Lehrpersonen (Fragestellung 2)}

Die Ergebnisse der MANOVA deuten darauf hin, dass die Sprachregion einen signifikanten und bedeutsamen Einfluss auf die sozialen Arbeitsbedingen von Schweizer Lehrpersonen hat, $V=0.21, F(6,512)=22.75, p \leq .001$, partial $\eta^{2}=$ .21. Das Geschlecht, $V=0.02, F(6,512)=1.78, p=.102$, partial $\eta^{2}=.02$, die Berufserfahrung, $V=0.05, F(18,1542)=1.52, p=.073$, partial $\eta^{2}=.02$, und die Schulgrösse (erfasst über die Anzahl Lehrpersonen an der Schule), $V=0.03$, $F(12,1026)=1.14, p=.322$, partial $\eta^{2}=.01$, hatten keinen Einfluss auf die sozialen Arbeitsbedingungen von Schweizer Lehrpersonen.

Im Rahmen von separaten t-Tests haben wir untersucht, hinsichtlich welcher Skalen sich die zwei Sprachregionen (Deutschschweiz vs. französisch- und italienischsprachige Schweiz) signifikant unterscheiden. Die Ergebnisse dieser Analysen sind in Tabelle 3 dargestellt. Als signifikant werden gemäss der Bonferroni-Korrektur Ergebnisse mit $p \leq .008$ (.05 / 6 Vergleiche) erachtet.

In Tabelle 3 ist ersichtlich, dass Lehrpersonen aus der Deutschschweiz die Unterstützung durch Schülerinnen und Schüler sowie durch Eltern signifikant 
und bedeutsam höher und somit positiver einschätzen als Lehrpersonen aus der französisch- und italienischsprachigen Schweiz.

Auch bei den gemeinsamen pädagogischen Vorstellungen haben die Lehrpersonen aus der Deutschschweiz einen signifikant höheren und somit positiveren Wert erzielt, dieser Effekt ist allerdings nicht bedeutsam $(d<.50)$. Schliesslich deuten die Ergebnisse der t-Tests darauf hin, dass Lehrpersonen aus der Deutschschweiz signifikant und bedeutsam weniger Konflikte mit Eltern erleben als ihre Kolleginnen und Kollegen aus der französisch- und italienischsprachigen Schweiz.

Tabelle 3: Mittelwerte (M) und Standardabweichungen (SD) der Lehrpersonen aus der Deutschschweiz (DS) $(N=420)$ und der Lehrpersonen aus der französisch- und italienischsprachigen Schweiz (FIS) $(N=166)$; Ergebnisse des Mittelwertvergleichs mittels $t$-Tests

\begin{tabular}{|c|c|c|c|c|c|c|}
\hline & \multicolumn{2}{|c|}{ DS } & \multicolumn{2}{|c|}{ FIS } & \multirow[b]{2}{*}{$t(\mathrm{df})$} & \multirow[b]{2}{*}{$d$} \\
\hline & $M$ & $S D$ & $M$ & $S D$ & & \\
\hline $\begin{array}{l}\text { Gemeinsame pädagogische } \\
\text { Vorstellungen }\end{array}$ & 3.46 & 0.76 & 3.12 & 0.78 & $4.88(579)^{*}$ & .45 \\
\hline Unterstützung durch Schüler & 3.80 & 0.54 & 3.30 & 0.63 & $8.85(262.19)^{*}$ & .84 \\
\hline Störungen im Unterricht & 2.59 & 0.68 & 2.57 & 0.69 & $0.27(581)$ & \\
\hline Unterstützung durch Eltern & 3.55 & 0.65 & 3.08 & 0.59 & $8.05(577)^{*}$ & .76 \\
\hline Konflikte mit Eltern & 2.11 & 0.73 & 2.63 & 0.91 & $-6.54(247.89)^{*}$ & .63 \\
\hline Führungsqualität & 3.56 & 0.76 & 3.58 & 0.85 & $-0.36(571)$ & \\
\hline
\end{tabular}

${ }^{*} p<.008$ (= gemäss Bonferroni angepasstes Signifikanz-Niveau: .05 / 6 Vergleiche)

\section{Diskussion}

Die sozialen Arbeitsbedingungen von Schweizer Lehrpersonen

Die Ergebnisse der Studie deuten darauf hin, dass Schweizer Lehrpersonen die sozialen Arbeitsbedingungen im Vergleich zu ihren Kolleginnen und Kollegen aus Baden-Württemberg positiver beurteilen. Die Belastung durch Störungen im Unterricht und Konflikte mit Eltern wurde von den Schweizer Lehrpersonen im Mittel als schwach bis mässig eingeschätzt. Störungen im Unterricht schätzten Schweizer Lehrpersonen dabei als weniger gravierend ein als Lehrpersonen aus Baden-Württemberg.

Gruppenvergleiche haben gezeigt, dass die sozialen Arbeitsbedingungen von Schweizer Lehrpersonen in Abhängigkeit von der Sprachregion mitunter unterschiedlich beurteilt werden. Lehrpersonen aus der französisch- und italienisch- 
sprachigen Schweiz nahmen weniger soziale Ressourcen (gemeinsame pädagogische Vorstellungen) und mehr soziale Fehlbelastungen (Konflikte mit Eltern) wahr als ihre Kolleginnen und Kollegen aus der Deutschschweiz. Umso ratsamer erscheint es, anhand aktuellerer Daten an Schulen in der französisch- und italienischsprachigen Schweiz zu prüfen, ob Handlungsbedarf zur Förderung sozialer Ressourcen besteht.

Überraschend war der fehlende Einfluss der Schulgrösse auf soziale Arbeitsbedingungen, da in internationalen Studien mit zunehmender Schulgrösse eine Verschlechterung der sozialen Arbeitsbedingungen vorhergesagt wird, beispielsweise wird mit zunehmender Schulgrösse ein geringeres Ausmass gemeinsamer pädagogischer Vorstellungen erwartet (Nübling, Vomstein, Haug, Nübling \& Adiwidjaja, 2011).

Die Ergebnisse der vorliegenden Untersuchung haben Implikationen für die Aus- und Weiterbildung von Lehrpersonen. Sie deuten zum einen darauf hin, dass Schweizer Lehrpersonen im Rahmen ihrer Ausbildung bereits dazu befähigt werden, vorhandene Ressourcen zu nutzen, um den hohen sozialen Anforderungen im Schulalltag begegnen zu können und dies auch als Komponente ihrer professionellen Selbstregulationsfähigkeit zu verstehen (Rothland, 2013c). Diese Ressourcen gilt es in Anbetracht künftiger Anforderungen an den Lehrerberuf (z.B. auch im Rahmen der Harmonisierung der Lehrpläne im Zuge der Einführung des «Lehrplan 21») weiter im Blick zu behalten.

In den Bildungsreformen der letzten zwei Jahrzehnte wurde die Bedeutung von Schulleitung sowie von Teamarbeit an den Schweizer Schulen gestärkt. Vor diesem Hintergrund scheinen gute Rahmenbedingungen für eine weitere Verbesserung der sozialen Arbeitsbedingungen zu bestehen. Schulleitungen werden zunehmend auf ihre Führungsaufgaben vorbereitet und die Gestaltung motivierender und gesundheitsförderlicher Arbeitsbedingungen wird in den entsprechenden Curricula thematisiert. Auch die Gestaltung eines guten Unterrichts unter Berücksichtigung der Lehrer-Schüler-Beziehungsqualität ist für viele Lehrpersonen selbstverständlicher Bestandteil von Aus- und Weiterbildungen. Eltern werden über Absprachen, Elternverträge o.ä. eingebunden, da die Erkenntnis an Schulen wächst, dass Schulen nur über ein Einbinden der Eltern erfolgreich sein können (Neuenschwander, 2009). Hier tut sich bereits viel. Besondere Beachtung sollte unseres Erachtens die Qualität der sozialen Beziehungen innerhalb des Schulkollegiums erhalten. Zwar werden diese sozialen Beziehungen an Schweizer Schulen bereits überwiegend positiv beurteilt. Allerdings wird möglicherweise gerade deshalb an Schulen übersehen, wie bedeutsam die Weiterentwicklung ist. So zeigen Trumpa et al. (2016) auf, dass ein verändertes Berufsverständnis notwendig wird, welches beispielsweise fest installierte Klassen-, Fach- oder Jahrgangsteams, gemeinsame Besprechungszeiten und obligatorische Anwesenheitszeiten in der Schule sowie regelmässige Supervision der Teambildungsprozesse beinhaltet. Genau an dieser Stelle ist nun auch Widerstand zu erwarten, da dies bei Lehrpersonen erstens als Eingriff in 
ihre Autonomie erlebt werden kann und zweitens die quantitative bzw. zeitliche Belastung kurzfristig ausdehnt. Erhellend sind an dieser Stelle die Studienergebnisse von Morgenroth (2015) hervorzuheben, die gut zu früheren Überlegungen von Stegmann (2008) passen. Sie zeigen die Ambivalenz der Lehrerkooperation auf, da von Lehrpersonen sowohl zeitliche Mehrbelastung und Einschränkung der Autonomie wie auch soziale Unterstützung und Entlastung berichtet werden. Eine entlastende Wirkung unter Wahrung der professionellen Autonomie stellt sich allerdings vor allem dann ein, wenn anspruchsvolle Formen der Kooperation (Ko-Konstruktion) gepflegt werden und eben nicht low-cost-Formen der Kooperation (Austausch, Synchronisation). Es lohnt sich somit, anspruchsvolle Formen der Kooperation in Lehrerteams an Schulen zu fördern, da sie als einzige Form der Kooperation eine im Hinblick auf die Belastungswahrnehmung entlastende Wirkung zu haben scheint und auch eine effektive Kooperationsform zur Unterstützung von Schulentwicklung darstellt (Morgenroth, 2015).

\section{Stärken, Einschränkungen und Ausblick}

Die Grundlage der vorliegenden Studie bildet eine landesweite Befragung von Schweizer Lehrpersonen. Das Vorgehen bei der Stichprobenselektion erlaubt es, gesamtschweizerische Aussagen zur Arbeitssituation von Klassenlehrpersonen zu machen und Teilgruppen von Schweizer Lehrpersonen hinsichtlich ihrer sozialen Belastungen miteinander zu vergleichen. Zudem konnten die Ergebnisse der RBSL-Studie mit Referenzwerten aus Baden-Württemberg verglichen werden.

Die folgenden Punkte müssen als Einschränkungen des vorliegenden Beitrages genannt werden: Erstens wurden sämtliche Angaben per Fragebogen erhoben und beruhen entsprechend auf Selbstauskünften. Für künftige Untersuchungen wäre es wünschenswert, diese Daten mit Fremdeinschätzungen zu ergänzen. Zweitens wies die einzige im Rahmen des SNF-Projekts für die vorliegende Studie neu entwickelte Skala (Unterstützung durch Schüler) eine unbefriedigende interne Konsistenz auf und sollte in der vorliegenden Version nicht mehr eingesetzt werden. Drittens umfasst ein vollständiges Bild der sozialen Arbeitssituation von Schweizer Lehrpersonen weitere Interaktionspartner, insbesondere eine Betrachtung zusätzlicher interner und externer Fachpersonen (z.B. Hausdienst, heilpädagogischer und schulpsychologischer Dienst, Sozialarbeitende), die in dieser Studie unberücksichtigt blieben. Viertens haben wir durch den Fokus auf die sozialen Arbeitsbedingungen andere potentielle Belastungen (z.B. Zeitdruck) bewusst ausgeklammert. Fünftens gab es zwar bemerkenswerte Unterschiede der Sprachregionen hinsichtlich der sozialen Arbeitsbedingungen. Diese Unterschiede können wir aber basierend auf der vorliegenden Studie noch nicht überzeugend erklären. Vielmehr wären differenzierte Analysen für einzelne Kantone oder gar Gemeinden notwendig, um die Besonderheiten etwa von Schulreformen und Schulfinanzierung angemessen berücksichtigen zu können. Derartige Analysen sind mit dem vorliegenden Datensatz nicht möglich, da die 
Stichprobengrösse nicht ausreicht, um Aussagen über einzelne Kantone treffen zu können.

\section{Schlussfolgerung}

Gesundheitsförderliche und motivierende Arbeitsbedingungen an Schulen sind zunächst natürlich im Interesse der Lehrpersonen selbst. Nach wie vor gehören Lehrpersonen so wie Angehörige anderer sozialer Berufe zu den stärker belasteten Arbeitsgruppen (Cramer et al., 2014). Daneben sind gute Arbeitsbedingungen im Interesse der Gesellschaft zu fördern, denn nur gesunde und motivierte Lehrpersonen können die geforderten hohen Bildungsstandards gewährleisten. Die regelmäßige Erhebung der Arbeitsbedingungen bei Lehrpersonen stellt die Grundlage eines systematischen nationalen Monitoring-Systems dar, im Rahmen dessen die Arbeitssituation von Lehrpersonen regelmäßig beurteilt und bei Bedarf Maßnahmen zu deren Optimierung abgeleitet werden können. Entsprechende Massnahmen gilt es im Rahmen der Aus- und Weiterbildung von Lehrpersonen zu berücksichtigen, um die Zielgruppen zu einem gesundheitsförderlichen Umgang mit Belastungen zu befähigen bzw. bei einer gezielten Nutzung und Aktivierung von Ressourcen zu unterstützen. Insbesondere auch Schulleitungen spielen hier eine zentrale Rolle, denn eine gesundheitsförderliche Gestaltung von Schulentwicklungsprojekten wird in geleiteten Schulen als eine Aufgabe der Schulleitung angesehen. Schulleitungen können im Rahmen der Aus- und Weiterbildung auf diese Aufgabe vorbereitet werden, um ihrerseits Schulteams zur Entwicklung anspruchsvoller Kooperationsformen (Ko-Konstruktion) zu befördern, die Entlastung unter Wahrung der professionellen Identität ermöglichen.

\section{Literatur}

Abdi, H. (2007). Bonferroni and Sidak corrections for multiple comparisons. In N. J. Salkind (Hrsg.), Encyclopedia of Measurement and Statistics. Thousand Oaks, CA: Sage. doi: $10.4135 / 9781412952644$

Antoniou, A. S., Polychroni, F. \& Vlachakis, A. N. (2006). Gender and age differences in occupational stress and professional burnout primary and between high-school teachers in Greece. Journal of Managerial Psychology, 21(7), 682-690.

Böhm-Kasper, O. (2004). Schulische Belastung und Beanspruchung. Münster: Waxmann.

Böhm-Kasper, O. \& Weishaupt, H. (2002). Belastung und Beanspruchung von Lehrern und Schülern am Gymnasium. Zeitschrift für Erziehungswissenschaft, 5(3), 472-499.

Cannata, M. (2007). Teacher community in elementary charter schools. Education Policy Analysis Archives, 15(11), 1-31.

Chaplain, R. P. (2008). Stress and psychological distress among trainee secondary teachers in England. Educational Psychology, 28(2), 195-209.

Cohen, J. (1988). Statistical Power Analysis for the Behavioral Sciences (2. Auflage). Hillsdale: Lawrence Erlbaum Associates. 
Cramer, C., Merk, S. \& Wesselborg, B. (2014). Psychische Erschöpfung von Lehrerinnen und Lehrern. Repräsentativer Berufsgruppenvergleich unter Kontrolle berufsspezifischer Merkmale. Lehrerbildung auf dem Prüfstand, 7(2), 138-156.

Dizinger, V., Fussangel, K. \& Böhm-Kasper, O. (2011). Lehrer/in sein an der Ganztagsschule: Neue Kooperationsanforderungen - neue Belastungen? Zeitschrift für Erziehungswissenschaft, 14(Suppl. 3), 43-61.

Forneck, H. J. \& Schriever, F. (2000). Die individualisierte Profession. Untersuchung der Lehrer/-innenarbeitszeit und-belastung im Kanton Zürich. Zürich: AZZH.

Fussangel, K., Dizinger, V., Böhm-Kasper, O. \& Gräsel, C. (2010). Kooperation, Belastung und Beanspruchung von Lehrkräften an Halb- und Ganztagsschulen. Unterrichtswissenschaft, 38(1), 51-67.

Grayson, J. L. \& Alvarez, H. K. (2008). School climate factors relating to teacher burnout: A mediator model. Teaching and Teacher Education, 24, 1349-1363.

Gregersen, S., Zimber, A., Kuhnert, S. \& Nienhaus, A. (2011). Führungsverhalten und Gesundheit - zum Stand der Forschung. Das Gesundheitswesen, 73(1), 3-12.

Hair, J. F., Black, W .C., Babin, B. J. \& Anderson, R. E. (2014). Multivariate Data Analysis (new int. ed.). Harlow: Pearson Education.

Halfhide, T. (2009). Teamteaching. In S. Fürstenau \& M. Gomolla (Hrsg.), Migration und schulischer Wandel: Unterricht (S. 103-120). Wiesbaden: VS Verlag für Sozialwissenschaften.

Harazd, B., Gieske, M., Gerick, J. \& Rolff, H.-G. (2009). Lehrergesundheit und Schulleitung: Ergebnisse des Forschungsprojektes. In B. Harazd, M. Gieske \& H.-G. Rolff (Hrsg.), Gesundheitsmanagement in der Schule. Lehrergesundheit als neue Aufgabe der Schulleitung (S. 44-103). Köln: Wolters Kluwer.

Igic, I., Keller, A., Luder, L., Brunner, B., Wieser, S., Elfering, A. \& Semmer, N. (2015). Job-Stress-Index 2015. Kennzahlen zu psychischer Gesundheit und Stress bei Erwerbstätigen in der Schweiz. Bern: Gesundheitsförderung Schweiz.

Klassen, R. M. \& Chiu, M. M. (2010). Effects on teachers' self-efficacy and job satisfaction: Teacher gender, years of experience, and job stress. Journal of Educational Psychology, 102(3), 741-756.

Krause, A. (2004). Fragebogen zur Arbeitssituation an Schulen (FASS). Dokumentation der Skalen und Aussagen. Unveröffentlichtes Manual. Freiburg: Albert-Ludwigs-Universität.

Krause, A. \& Dorsemagen, C. (2011). Gesundheitsförderung für Lehrerinnen und Lehrer. In E. Bamberg, A. Ducki \& A.-M. Metz (Hrsg.), Gesundheitsförderung und Gesundheitsmanagement in der Arbeitswelt (S. 139-157). Göttingen: Hogrefe.

Laux, A. (2011). Schulleitung im Mittelpunkt schulischer Gesundheit: Eine Studie zu der Gesundheit schulischer Führungskräfte und ihrer Rolle für die Lehrergesundheit. Dissertation: Universität Potsdam.

Morgenroth, S. (2015). Lehrerkooperation unter Innovationsstress. Soziale Stressbewältigung als wertvoller Wegweiser. Wiesbaden: Springer VS.

Neuenschwander, M. P. (2009). Schule und Familie -Aufwachsen in einer heterogenen Umwelt. In H.-U. Grunder \& A. Gut (Hrsg.), Zum Umgang mit Heterogenität in der Schule (Band1, S. 148-168). Baltmannsweiler: Schneider Verlag.

Nübling, M., Vomstein, M., Haug, A., Nübling, T., \& Adiwidjaja, A. (2011). European-wide survey on teachers work related stress - Assessment, comparison and evaluation of the impact of psychosocial hazards on teachers at their workplace. Freiburg: Freiburg research centre occupational and social medicine.

Nübling, M., Vomstein, M., Haug, A., Nübling, T., Stössel, U., Hasselhorn, H.-M., Hofmann, F., Neuner, R., Wirtz, M. \& Krause, A. (2012). Befragung zu psychosozialen Faktoren am Arbeitsplatz. Personenbezogene Gefährdungsbeurteilung an öffentlichen Schulen in Baden-Württemberg. Freiburg: Freiburger Forschungsstelle Arbeits- und Sozialmedizin FFAS. 
Nübling, M., Wirtz, M., Neuner, R. \& Krause, A. (2008). Ermittlung psychischer Belastungen bei Lehrkräften - Entwicklung eines Instruments für die Vollerhebung in Baden-Württemberg. Zentralblatt für Arbeitsmedizin, Arbeitsschutz und Ergonomie, 58, 212-213.

Philipp, A. \& Kunter, M. (2013). How do teachers spend their time? A study on teachers' strategies of selection, optimisation, and compensation over their career cycle. Teaching and Teacher Education, 35(1), 1-12.

Rothland, M. (Hrsg) (2013a). Belastung und Beanspruchung im Lehrerberuf-Modelle, Befunde, Interventionen (2., vollständig überarbeitete Auflage). Wiesbaden: Springer VS.

Rothland, M. (2013b). Soziale Unterstützung. Bedeutung und Bedingungen im Lehrerberuf. In M. Rothland (Hrsg.), Belastung und Beanspruchung im Lehrerberuf-Modelle, Befunde, Interventionen (2., vollständig überarbeitete Auflage) (S. 231-250). Wiesbaden: Springer VS.

Rothland, M. (2013c). Beruf: Lehrer/Lehrerin - Arbeitsplatz: Schule. Charakteristika der Arbeitstätigkeit und Bedingungen der Berufssituation. In M. Rothland (Hrsg.), Belastung und Beanspruchung im Lehrerberuf - Modelle, Befunde, Interventionen (2., vollständig überarbeitete Auflage) (S. 21-39). Wiesbaden: Springer VS.

Rothland, M. \& Klusmann, U. (2012). Belastung und Beanspruchung im Lehrerberuf. In S. Rahm \& C. Nerowski (Hrsg.), Enzyklopädie Erziehungswissenschaft Online (EEO), Fachgebiet Schulpädagogik (S. 1-42). Weinheim: Juventa.

Sandmeier, A., Kunz Heim, D., Windlin, B. \& Krause, A. (2017). Negative Beanspruchung von Schweizer Lehrpersonen. Trends von 2006 bis 2014. Schweizerische Zeitschrift für Bildungswissenschaften, 39(1), 75-94.

Schaller, R. (2015). Helikoptereltern sind besonders anstrengend. ZLV Magazin, 5, 10-15.

Schult, J., Münzer-Schrobildgen, M. \& Sparfeldt, J. R. (2014). Belastet, aber hochzufrieden? Arbeitsbelastungen von Lehrkräften im Quer- und Längsschnitt. Zeitschrift für Gesundheitspsychologie, 22(2), 61-67.

Skaalvik, E. M. \& Skaalvik, S. (2007). Dimensions of teacher self-efficacy and relations with strain factors, perceived collective teacher efficacy, and teacher burnout. Journal of Educational Psychology, 99(3), 611-625.

Skaalvik, E. M. \& Skaalvik, S. (2010). Teacher self-efficacy and teacher burnout: A study of relations. Teaching and Teacher Education, 26(4), 1059-1069.

Stegmann, S. (2008). Einzelkämpfer oder Teamplayer? Soziale Arbeitsbedingungen an Schulen. In A. Krause, H. Schüpbach, E. Ulich \& M. Wülser (Hrsg.), Arbeitsort Schule - Organisations- und arbeitspsychologische Perspektiven (S. 365-386). Wiesbaden: Gabler.

Trumpa, S., Franz, E. \& Greiten, S. (2016). Forschungsbefunde zur Kooperation von Lehrkräften - Ein narratives Review. Die Deutsche Schule, 108(1), 82-94.

Vuille, J.-C., Carvajal, M. I., Casaulta, F. \& Schenkel, M. (2004). Die gesunde Schule im Umbruch. Wie eine Stadt versucht, eine Idee umzusetzen und was die Menschen davon spüren. Zürich: Verlag Rüegger.

\section{Schlagworte: Belastungen, Ressourcen}




\section{Les conditions sociales des enseignants Suisses - Résultats d'une étude nationale}

\section{Résumé}

Cet article, qui se base sur une enquête nationale, étudie les conditions sociales de travail des enseignants et enseignantes suisses ainsi que les différences potentielles provenant de l'influence de la démographie (genre, expérience professionnelle et région linguistique) et de la grandeur des écoles. Les résultats signalent que les enseignants suisses évaluent leurs conditions sociales de travail de manière majoritairement positive comparé à un sondage réalisé en Allemagne. Concernant les régions linguistiques où se situent les écoles, l'étude met en évidence des différences significatives. Les résultats sont discutés en vue de la formation de base et de la formation continue des enseignants suisses.

Mots-clés: Charges de travail, ressources

\section{Le condizioni sociali di lavoro degli insegnanti svizzeri - Risultati di uno studio nazionale}

Riassunto

Nell'ambito del presente contributo, tramite un sondaggio effettuato su scala nazionale, sono state esaminate le condizioni sociali di lavoro degli insegnanti svizzeri, così come potenziali differenze demografiche (sesso, esperienza di lavoro e regione linguistica) e inerenti alla grandezza della scuola. I risultati mostrano che gli insegnanti svizzeri tendono a valutare in modo più positivo le loro condizioni sociali di lavoro rispetto ad un gruppo di confronto tedesco. Riguardo alla regione linguistica della scuola, sono state trovate differenze significative. I risultati della ricerca sono di seguito discussi in vista della formazione e dell'aggiornamento degli insegnanti svizzeri.

Parole chiave: Incarichi, risorse 


\section{Social working conditions of Swiss teachers - Findings of a national study}

Based on a national survey, the present article considers social working conditions of Swiss teachers and estimates the effects of demography (sex, work experience, language region) and school size on the evaluation of social demands and resources. Results indicate that Swiss teachers evaluate their social working environment predominantly positive compared to a sample of German teachers. There were significant differences with respect to language regions. The findings are discussed in reference to training and development of Swiss teachers.

Keywords: Demands, resources 